\title{
Building a More Diverse and Inclusive Science: Mindfulness-Based Approaches for Latinx Individuals
}

\author{
Gabriela A. Nagy ${ }^{1,2}$ (C) Criss Cuervo 3,4 . Elaine Y. Ramos Rodríguez ${ }^{3,4} \cdot$ Jennifer Plumb Vilardaga ${ }^{1} \cdot$ Noga Zerubavel $^{1}$. \\ Jessica L. West ${ }^{1} \cdot$ Michael C. Falick ${ }^{4,5}$. Diana C. Parra ${ }^{6}$
}

Accepted: 15 February 2022 / Published online: 8 March 2022

(c) The Author(s), under exclusive licence to Springer Science+Business Media, LLC, part of Springer Nature 2022

\begin{abstract}
Objectives This article represents a call to action for the mindfulness field to be more diverse and inclusive of Latinx individuals. Building a diverse and inclusive science around mindfulness-based approaches (MBAs) that considers important group-level cultural and contextual information is an important public health challenge in need of innovative solutions.

Methods We describe ways that the Latinx population is poised to benefit from MBAs. We further elucidate challenges, describe potential solutions, and outline a research agenda that may hold promise for building a more inclusive mindfulness movement.

Results Our recommendations center around developing nuanced cultural adaptations to MBAs, engaging Latinx individuals in research, increasing the rigor of scientific studies pertaining to Latinx individuals, relying on implementation science to develop innovative methods for disseminating MBAs to Latinx individuals, developing training and certification mechanisms to increase diversity and representation of Latinx mindfulness teachers, and creating mechanisms for the oversight of MBAs within this group.

Conclusions There has been a lack of inclusivity of Latinx individuals in the field of MBAs with regards to research studies, barriers to access for economically disadvantaged groups, and lack of diversity in its workforce. Considering the recognition of adverse social drivers of health that generate chronic stress and health disparities, the Latinx population is especially poised to benefit greatly from MBAs. A diverse and inclusive mindfulness science holds promise to enhance the effectiveness, acceptability, feasibility, and wide-scale dissemination and implementation of MBAs.
\end{abstract}

Keywords Mindfulness $\cdot$ Meditation $\cdot$ Latinx $\cdot$ Immigrants $\cdot$ Acculturative stress $\cdot$ Implementation science $\cdot$ Cultural adaptations $\cdot$ Diversity and inclusion

Latinx (gender-inclusive term for individuals of Latin American descent; interchangeable with Latino, Latina, Latin

Gabriela A. Nagy

gabriela.nagy@duke.edu

1 Department of Psychiatry and Behavioral Sciences, Duke University School of Medicine, Duke University Medical Center, DUMC 3026, Durham, NC 27710, USA

2 Duke University School of Nursing, Durham, NC, USA

3 Alma Y Espíritu, Chicago, IL, USA

4 Mindfulness Studies, Lesley University, Cambridge, MA, USA

5 Rothfelder \& Falick, L.L.P., Houston, TX, USA

6 Prevention Research Center, Brown School Washington University in St. Louis, St. Louis, MO, USA
American, and Hispanic) individuals represent the largest and second-fastest growing racial/ethnic minoritized group in the United States (US), comprising $18.5 \%$ of the total US population ( 60 million), of which two-thirds are US-born ( 40 million) and one-third were born in a Latin American country ( 20 million; Noe-Bustamante \& Flores, 2019). The increasingly growing Latinx population is projected to comprise $29 \%$ of the total US population by 2065 (107 million; Passel \& Cohn, 2008). Herein, we focus on the overall Latinx population, inclusive of immigrants and US-born individuals.

In the literature, there is often a one-story narrative focused on ways in which Latinx communities are "vulnerable" or "at risk," which perpetuates further marginalization of this population. To offset this, we highlight the wealth of cultural strengths and resilience factors that 
have also been noted in the literature that are protective for the health of Latinx individuals at various levels (Cardoso \& Thompson, 2010; Gallo et al., 2009). As noted by Cardoso and Thompson (2010), at the individual level, Latinx immigrants may possess a combination of biological and psychosocial factors that can shape resilience before, during, and after the migration journey (e.g., temperament, intelligence, social competence, self-regulation, self-esteem, self-mastery, and self-efficacy, among others). They also note that family strengths (i.e., familism) play a vital role in shaping the resilience of Latinx immigrants. Familism refers to kinship networks marked by support, closeness, connectedness, loyalty, respect, solidarity, and interdependence. Cultural factors (such as those emphasizing strong personal connections and levity [simpatia/ friendliness] and a collectivist orientation and prioritizing other people over oneself [allocentrism]) have also been described as resilience factors that can be protective to the health of Latinx immigrants by reinforcing ethnic and cultural pride, generating a sense of connectedness and solidarity, and weakening the impact of acculturative stress (Cardoso \& Thompson, 2010). Extended community support, such as those found in neighborhoods, religious communities, and schools, has also been described as a unique resilience factor for Latinx immigrants (Cardoso \& Thompson, 2010).

In spite of the fact that they represent a sizable segment of the population and hold a range of resilience factors as noted above, Latinx individuals face a myriad of adversities that lead to further marginalization, having a negative impact on their health (Lopez et al., 2018). In fact, there is research to show that the longer Latinx individuals live in the US, the more their health is eroded (Cho et al., 2004). Latinx individuals disproportionately face unfavorable social conditions that have an adverse effect on mental and physical health, such as lower socioeconomic status, lower access to employment opportunities that would allow economic mobility, increased likelihood of having high-risk/ low-compensation employment, higher unemployment rates, lower educational attainment, residential segregation, and lack of access to adequate healthcare, among others (Do et al., 2017; Duncan et al., 2006; Gany et al., 2014; Ramirez \& de la Cruz, 2003; Reimers, 2006). These factors are known as adverse social drivers of health. These structural factors contribute to the disproportionate emergence of some health conditions and poor quality of life that have less to do with biological factors and more to do with the social, economic, and physical structures in which marginalized groups of people live, work, and play (Braveman \& Gruskin, 2003). The most recent example of how adverse social drivers of health have implications for wellbeing of marginalized communities is the disproportionate burden of COVID-19 on Latinx individuals in the US (Holden et al., 2021; Macias
Gil et al., 2020), which compound existing disparities facing this population (Vega et al., 2009).

One important finding regarding the contexts that shape the wellbeing of Latinx individuals is the high exposure to traumatic events (i.e., direct or indirect exposure to an event that involves actual or threatened death, or serious injury, or sexual violence to oneself or another person; APA, 2013). Traumatic stress is a profound experience of stress in response to an intense threat that manifests in physical and psychological processes that extend beyond the traumatic event/s (Gerhart et al., 2015; Treleaven, 2018). Of particular salience for this population may be the traumatic experiences occurring in childhood, which are referred to as adverse childhood experiences (ACEs; Caballero et al., 2017), as well as those related to migration (Perreira \& Ornelas, 2013). Importantly, it has been noted that the existing literature may underestimate the prevalence of ACEs in Latinx immigrants because they do not adequately capture potentially traumatic immigrant experiences (Caballero et al., 2017). Indeed, some recent work in a sample of young adult Latinx immigrants found rates of ACEs higher than previously reported in the literature (Bravo et al., 2021).

Additionally, traumatic experiences can at times be precipitants to migration and/or displacement (e.g., state-sanctioned violence, gang violence, humanitarian crises, natural disasters, intimate partner, and familial violence). In addition to the aforementioned adverse social drivers of health, they endure the additional challenges that can come from their unique experience of migration, known as acculturative stress. Acculturative stress refers to adversities faced by immigrants and refugees before, during, and after the process of migration and can arise from the simultaneous pressure to maintain aspects of one's culture while also adopting aspects of the mainstream culture in their new environment-a process known as acculturation (Berry, 2003). Stated another way, acculturative stress is the psychological adjustment that occurs when culturally distinct groups and individuals come into contact with another culture (Berry \& Kim, 1988). Acculturative stressors can include, but are not limited to, pre-migration circumstances (e.g., a lack of economic and educational opportunities, adverse childhood experiences, and experiences and/or threats of physical and sexual violence, extortion, gang activity, and organized crime), traumatic migration journeys (e.g., experiencing injury and/or sexual violence, witnessing death), language barriers, negotiating differing and sometimes conflicting cultural values (i.e., intergenerational gaps), navigating between American and Latinx ways of daily living, assimilation pressures, separation from family and/or intergenerational family conflict, and discrimination (Cervantes et al., 2016). At lower frequencies or intensities, these adversities may be experienced as stressful, but at their most extreme levels, they may be experienced as traumatic. Importantly, 
acculturative stress has been identified as one of the most robust predictors of health decline in Latinx individuals (Gonzalez-Guarda et al., 2021).

The chronic and compounding nature of the upstream stressors negatively impacts the health and wellbeing of this population. Unfortunately, the result of these factors is that many Latinx individuals are at risk for downstream problems such as anxiety, mood disruption, trauma-related responding, substance misuse, and relationship conflict that can impact daily functioning (Alegria et al., 2006; Alvarez et al., 2007; Cummings et al., 2013). Access to mental health care is therefore extremely important for individuals coping with traumatic stress, acculturative stress, and resulting mental health challenges. Unfortunately, as noted by Guarnaccia et al. (2005), despite significant acculturative stress and mental health challenges, Latinx individuals face a range of barriers at the individual, community, service system, and broader societal levels to accessing mental health care. There thus is recognition of the need to identify strategies that effectively engage Latinxs with approaches that could improve emotional and behavioral wellbeing (Guarnaccia et al., 2005).

Latinx individuals are poised to benefit from approaches that can improve health, wellbeing, and resilience. Mindfulness-based approaches (MBAs) hold such promise. However, despite the dramatic increase of mindfulness in psychotherapy, consumer apps, print media, social media, videos, audio files, and podcasts, one consistent observation is that Latinx individuals have not been well-represented as practitioners, researchers, clinicians, or instructors of mindfulness. In addition, there has been a lack of empirical inquiry related to how MBAs could reduce the impact of the unique configuration of stressors facing Latinx individuals (e.g., traumatic stress and acculturative stress). Herein, we discuss why Latinx individuals residing in the US are especially positioned to take advantage of what mindfulness has to offer. We elucidate challenges facing the field of mindfulness, especially as it relates to extending mindfulness to Latinx individuals. Lastly, we provide a list of strategies to build a diverse and inclusive mindfulness-based science.

\section{MBAs Hold Promise for Improving the Health of Latinx Individuals}

Mindfulness-based approaches refer to those approaches that stem from attentional practices, open monitoring practices, and compassion-focused practices, as well as the processes and characteristics of mindfulness used within the scientific and medical community, often in the context of programs integrating MBAs. MBAs have demonstrated repeated and robust effectiveness across the lifespan on a number of important psychological (Boyd et al., 2018;
Hofmann, \& Gómez, 2017) and physical health outcomes (Black \& Slavich, 2016; Hilton et al., 2017) in the general population. Given this array of helpful and relevant benefits, MBAs are poised to support the health of Latinx individuals in important ways. For example, Cotter and Jones' (2020) systematic review examining the feasibility and efficacy of MBAs in Latinx participants described potential promising effects on stress and other health outcomes using diverse MBAs. However, they noted important limitations of the extant scientific knowledge base-the scarcity of research in this area; in particular, the paucity of randomized controlled trials which limit the ability to infer causation; the limited use of cultural adaptations (such as the use of Spanish-language materials or a Latinx facilitator); the use of small sample sizes and a lack of rigorous research designs; the lack of longterm tracking of health-related outcomes post-intervention and longer-term follow-up; and the fact that most studies in this review treated the Latinx participants as a single group rather than conducting sub-group analyses recognizing this is a vastly heterogeneous population.

Consistent with these conclusions, Castellanos et al.'s (2020) systematic review and meta-analysis exploring the effectiveness of cultural adaptations of MBAs for Latinx individuals noted that culturally adapted MBAs were associated with stress reduction as well as improvement in depressive symptoms, stress management, and chronic illness management. Furthermore, MBAs resulted in a moderate-to-large effect of the interventions on psychiatric distress relative to scores in the comparison group. Nonetheless, the authors emphasized that the small body of work available limited their ability to make meaningful conclusions as to the effectiveness of MBAs for Latinx individuals and the cultural adaptations that may enhance engagement in this population.

Importantly, existing findings of MBAs and Latinx individuals indicate improvements in health-related quality of life (Roth \& Stanley, 2002; Vinci et al., 2020); mindful attention awareness, self-compassion, perceived stress, depression (Edwards et al., 2014); life satisfaction (Neece, 2014); and reduced post-traumatic stress disorder (PTSD) symptoms (Viana et al., 2017); and highlight the importance of mindful eating to facilitate healthy eating patterns and meal planning among Latinas with disordered eating behaviors (Reyes-Rodríguez et al., 2016). Despite the potential benefit of MBAs for Latinx individuals, some scholars have found that minoritized populations, such as Latinxs, are less likely to engage in mindfulness practices and are thereby less likely to receive potential health benefits (Olano et al., 2015). These data highlight the need for the mindfulness field to prioritize strategies for better meeting the needs of minoritized groups. 


\section{The Use of MBAs to Reduce Stress in Latinx Individuals}

One of the most pervasive topics of research on MBAs is about its impact on reducing stress (e.g., Khoury et al., 2015; Sharma \& Rush, 2014). The potential impact of mindfulness on stress in Latinx individuals has been studied in the literature, with studies showing decreases in perceived stress as a function of mindfulness (e.g., LopezMaya et al., 2019). Herein, we discuss the application of MBAs to intervene on common stressors faced by Latinx individuals, namely traumatic stress and acculturative stress. We acknowledge that there are a range of stressors aside from these that may be relevant for Latinx individuals; we nonetheless limit our discussion to these as our anecdotal experience indicates these are two areas that represent important targets for intervention that could be targeted by MBAs.

\section{Reducing Traumatic Stress with MBAs}

Inclusive understandings of traumatic stress allow increased multicultural sensitivity by incorporating racial/ethnic trauma (Chavez-Dueñas et al., 2019), historical trauma (Estrada, 2009), cultural trauma (Lehrner $\&$ Yehuda, 2018), and intergenerational trauma (Cerdeña et al., 2021). That is, using frameworks that conceptualize racism and ethnoviolence as trauma (Bryant-Davis \& Ocampo, 2005) allows clinicians and other MBA facilitators to more effectively implement trauma-related assessment and interventions in a culturally responsive manner (Helms et al., 2012). Furthermore, the intergenerational transmission of trauma through mechanisms including in utero exposure to stress hormones, post-natal accommodation to parental behavior, and epigenetic transmission has been well-established (Lehrner \& Yehuda, 2018; Yehuda \& Lerner, 2018), demonstrating that the legacy of trauma continues across generations. Therefore, if family members faced trauma and violence prior to migration, during migration, or post-migration, the future generations of this family may inherit some of the family's adaptations to traumatic stress (i.e., symptoms). For example, a parent with PTSD or dissociative symptoms associated with traumatic experiences in a violent context that they fled may teach their child to be suspicious and distrusting of others or may be inadvertently inattentive to the child's emotional needs due to impairment from their symptoms.

We posit that MBAs are well-suited to address trauma in Latinx immigrants caused in part by systemic oppression, immigration journeys, and the resettlement process (Rettger et al., 2016). There is evidence to support the use of several MBAs for individuals with complex PTSD as useful for a variety of trauma-related symptoms (e.g., avoidance, affect dysregulation, behavioral dysregulation, attention dysregulation, dissociation, and identity disturbance), including mindfulness-based stress reduction (MBSR; Polusny et al., 2015), mindfulness-based cognitive therapy (MBCT; King et al., 2013), and compassion meditation (Hinton et al., 2013; Kearney et al., 2013), among others. Indeed, recent clinical guidelines from the International Society for Traumatic Stress Studies include many MBAs among their list of recommended treatments for trauma-related problems (Bisson et al., 2019). Metaanalytic reviews suggest that MBAs for trauma survivors are effective in reducing post-traumatic symptoms and increasing mindfulness compared to control conditions (Gallegos et al., 2017; Hopwood \& Schutte, 2017). These studies also suggest that longer mindfulness interventions have greater efficacy in reducing symptoms.

Mindfulness can help trauma survivors in a number of ways. First and foremost, trauma survivors often re-experience events from the past or have frequent memories of trauma, and therefore building skills to stay centered in the present moment is clinically helpful (Boyd et al., 2018). The attentional training involved in mindfulness is theorized to be central in this regard (Boyd et al., 2018; Holzel et al., 2011; Lang et al., 2012). Mindfulness facilitates exposure, extinction, and reconsolidation in response to emotions (Holzel et al., 2011). Fear may be a particularly critical target for emotional exposure in the context of PTSD (Kummar, 2018). This process can also be applied to other traumarelated emotions including guilt, shame, disgust, anger, and sadness. Mindfulness cultivates cognitive reappraisal, allowing individuals to see things differently and facilitate emotion regulation (Holzel et al., 2011). In treatments for trauma, acceptance is a centrally emphasized reappraisal process, and in MBAs, a key component is non-judgmental acceptance of emotions (Lang et al., 2012) as well as coping with memories of the past (Meyer et al., 2018). In addition, mindfulness is theorized to target dissociation directly by providing an alternative method of cognitive detachment (Forner, 2019). Finally, compassion-focused meditation (e.g., metta/loving-kindness) appears to enhance emotional flexibility by increasing trauma survivors' ability to engage positive emotions (Hinton et al., 2013; Kearney et al., 2013). Compassion-focused meditation has been theorized to be particularly important for traumatized minoritized groups due to its ability to facilitate cognitive and emotional flexibility (Hinton et al., 2013).

Numerous studies have established the effectiveness of mindfulness-based interventions in decreasing distress and symptoms associated with traumatic stress (Hopwood $\&$ Schutte, 2017) and recommendations on bringing a trauma-informed lens to mindfulness training are well 
delineated (see King \& Favorite, 2016; Treleaven, 2018; and Waelde, 2021). Finally, mindfulness can strengthen resilience in the face of adversity, thereby helping individuals buffer the impact of stress and trauma (Goyal et al., 2014; Southwick et al., 2015). In fact, mindfulness and acceptance have been conceptualized as key aspects of resilience following exposure to trauma (Thompson et al., 2011). Thus, mindfulness can function as an intervention and treatment for symptoms that have arisen in response to stress and trauma and can simultaneously be resilience enhancing to buffer against future adversity.

\section{Reducing Acculturative Stress with MBAs}

Given the clear benefits of mindfulness on stress and the extensive stressors that are encountered during the immigration and acculturation process, MBAs may offer a relevant and effective theoretical and philosophical model that could aid in buffering acculturative stress. We propose that mindfulness could help individuals become more non-judgmentally aware of the differences between cultures (e.g., the one from their country of origin and the one in their receiving country), and the associated emotions that arise with these differences without evaluating them as good or bad. Holding these in awareness with non-judgment while practicing compassionate acceptance may reduce some of the associated stress that comes with acculturation. Furthermore, mindfulness could aid in stress reduction via increasing values clarification (e.g., where there are values conflicts, as is often common between parents and children) and contributing to effective listening and communicating in the setting of intergenerational interpersonal conflict. There is emerging empirical support indicating mindfulness is associated with less acculturative stress. For example, a recent study demonstrated preliminary evidence that dispositional mindfulness is associated with less acculturative stress and negative automatic thoughts in Latinx youth (Schlaudt et al., 2021).

\section{Recommendations for Building a Latinx Inclusive Mindfulness Science}

Informed by our review of the literature as well as our experience as mindfulness practitioners, researchers, clinicians, and instructors, we delineate a set of recommendations that will allow the mindfulness movement to have a wider reach, taking into account the unique experiences and characteristics of the Latinx population residing in the US. Doing so can increase the generalizability of research findings and further enhance science in this area.

\section{Recognize the Vast Heterogeneity Within the Latinx Population to Increase Fit}

Any discussion on increasing diversity and inclusiveness in the field of mindfulness would be incomplete without acknowledging the vast heterogeneity located within the Latinx population as well as the intersection of distinct experiences, identities, and systems of privilege and oppression affecting this population. Latin America comprises many Spanish-speaking countries with a diversity of races (e.g., Black, White); ancestral backgrounds (e.g., indigenous, African diaspora, Spanish); cultures (i.e., traditions and customs); socioeconomic statuses; education levels; and languages, dialects, and idioms and living conditions. Furthermore, Latinx immigrants evidence variability in migration trajectories (e.g., by foot, plane, or boat), circumstances of exit and degree of planning around their migration (e.g., forced migration, planned migration), reasons for migration (e.g., fleeing violence in their home country, search for better educational opportunities, to reunite with family), conditions during migration, documentation status (e.g., US citizen, permanent resident, visa holder, undocumented), receiving communities (e.g., established ethnic enclaves vs. emerging diffuse communities), and access to health care (Ackert et al., 2021). We thus recommend that mindfulness researchers, clinicians, and instructors carefully assess relevant sociodemographic, cultural, and contextual factors of the individuals with which they are working to ensure that the content presented is deemed to be relevant and appropriate for the specific group they aim to reach. We encourage particular attention to acculturative stress and traumatic stress as particularly relevant contextual factors, as previously noted, to culturally adapt MBAs.

Culturally adapted interventions are more effective than unadapted interventions for minoritized groups, including Latinxs (Benish et al., 2011). Adaptation of interventions can also improve implementation outcomes, such as acceptability and appropriateness in minoritized populations (Cabassa \& Baumann, 2013). In recent years, various independent research groups have taken steps to develop and test cultural adaptations to MBAs for Latinx individuals. A meta-analysis by Cotter and Jones (2020) described cultural adaptations at various levels across the studies they reviewed. To address barriers to engagement, adaptations have centered on problem-solving transportation problems, offering childcare, providing the intervention at a low cost or free-of-charge, and scheduling sessions at convenient times. Adaptations to the intervention content have included changing the language (to Spanish), ensuring materials are at an appropriate reading level, holding sessions more frequently, and inclusion of culturally responsive emotion regulation techniques. Some interventions have relied on community-engaged methods to 
partner with community agencies to align the mindfulness intervention with community needs. Lastly, they found that some interventions worked toward increasing buy-in for the MBAs by including testimonials to enhance perceptions of credibility.

A meta-analysis by Castellanos et al. (2020) also noted similar types of adaptations. Namely, language adaptations referred to utilization of culturally appropriate language for the person/group. Furthermore, they noted that merely translating materials did not in itself constitute cultural adaptation. Metaphor adaptations pertained to the inclusion of sayings or stories from the culture of participants' own stories and metaphors. Method adaptations were conducted to intentionally modify materials to fit the intended culture yet doing so without sacrificing methodological rigor. Goal adaptations refer to treatment goals that include cultural adaptations with regard to participants' values, customs, or traditions. Concept adaptations focused on the explicit integration of cultural aspects into the conceptualization of the psychological model and the theorized process. We thus recommend that when attempting to utilize MBAs with Latinx individuals, teams carefully assess whether adaptations are warranted for use with the specific group with which they are working to maximize the intervention's fit, and then to test and incorporate targeted adaptations.

\section{Utilize a Strength-Based Approach}

As mentioned earlier, Latinx immigrants possess a range of innate resilience factors that are protective of health at various levels. Thus, framing MBAs to capitalize on these factors can be essential to the effectiveness of interventions among Latinx. In particular, social networks and family strength may act as protective resilience factors. Positive peer relationships located through these networks for Latinx immigrants can offer different kinds of support (e.g., emotional, instrumental) and foster connectedness in the face of negative experiences that are especially salient for immigrants. Importantly, it has been noted that individuals who lack this kind of social support may be more vulnerable to discrimination and related experiences. Thus, we recommend that adaptations of MBAs for the Latinx population take into consideration the power of community, family, and social relationships. Note that this may be a point of divergence from mainstream mindfulness-based teachings, which typically place higher importance on individual versus community practice. It is our recommendation to consider the existing resilience factors present in Latinx individuals to help strengthen adherence, efficacy, and overall equip participants with tools that are aligned with already existing resilience factors.

\section{Incorporate Religious Content into MBAs When Pertinent}

One barrier to Latinx engagement in MBAs may be the historical roots of mindfulness, as mindfulness practices have been most systematically elaborated in the Buddhist religion and most Western medicine interventions were developed as secular adaptations of this tradition. Specifically, some scholars note a potential for incongruence between Christian religious beliefs prevalent in some Latinx communities and Eastern mindfulness traditions (Cotter \& Jones, 2020; Ortiz et al., 2019). In our experience, it is common for participants in MBAs to have questions about the relationship between mindfulness and religious practices; therefore, clinicians and instructors need to be prepared to respond to such concerns, as well as to provide integrative frameworks to facilitate a complementary relationship between mindfulness and religion.

Although Buddhism has elaborated detailed descriptions of mindfulness practices, contemplative practices are cornerstone elements of most religious traditions, including Christianity (Kabat-Zinn, 2005). The most highly regarded Buddhist religious leader notes that if Buddhist "methods can be shown through scientific tests to be of benefit, then there is every reason to find ways to make them available to everyone, whether or not they are interested in Buddhism itself" (foreword by His Holiness the Fourteenth Dalai Lama in Goleman, 2003, p. xiv). In fact, most therapeutic interventions use a secular theoretical and philosophical framework rather than a religious framework (e.g., Grossman \& Van Dam, 2011; Kabat-Zinn, 2005; Segal et al., 2018). We thus recommend that in developing or adapting MBAs for Latinxs, researchers, clinicians, and instructors would benefit from considering participants' religious beliefs that many associate with mindfulness. For example, a religious practice of giving one's stressors "over to God" may be cast as a form of acceptance, and prayer could be discussed as a form of contemplation and observation of experience akin to mindfulness. Specifically, some cultural adaptations may include the integration of religious themes, iconography, or practices, such as praying the rosary. In addition, a culturally responsive adaptation may be helpful to provide explicit psychoeducation dispelling myths about mindfulness (Cotter \& Jones, 2020).

\section{Increase Representation of Latinx Individuals in Mindfulness-Based Research}

Latinx individuals are underrepresented in research studies on MBAs (Castellanos et al., 2020; Chin et al., 2019; Cotter \& Jones, 2020; DeLuca et al., 2018). For example, Waldron et al. (2018) found that across randomized controlled trial studies $(N=69)$, Latinx individuals represented only 
$4 \%$ of total participants. As noted in a systematic review by DeLuca and colleagues (DeLuca et al., 2018), there are several factors that may impede the inclusion of Latinx individuals in mindfulness-based science, including healthcare disparities, historical underrepresentation in clinical research, and a conceptual perspective that emphasizes the universality of Buddhist teachings. Considering that lack of representation in mindfulness research can pose a threat to the generalizability of findings and impact, we recommend that researchers take deliberate and intentional steps to engage this population in research. As noted by several researchers focused on innovating methods for increasing representation of minoritized groups in research, these strategies can include community-engaged strategies, snowballing sampling methods, direct contact with participants, and using bilingual and bicultural research staff (García et al., 2017; Mier et al., 2006; Nápoles-Springer et al., 2000).

\section{Build a More Representative Mindfulness Workforce}

To our knowledge, there have not been published reports of the representation in the mindfulness workforce by race and ethnicity. As a proxy, there is data to indicate that only $5 \%$ of the total psychology workforce comprises Latinx individuals (Lin et al., 2018). To address the significant lack of diversity, there have been efforts by organizations and academic institutions to increase training opportunities in MBAs for diverse and underrepresented communities. Notably, the Mindfulness-Based Professional Training Institute at UC San Diego Center for Mindfulness received a grant to provide training and scholarships to members of the community in MBSR teacher training (MindfulnessBased Professional Training Institute, 2020). Unfortunately, efforts such as these ones are not sufficient. Increasing representation in the mindfulness workforce, therefore, requires comprehensive, deliberate, and sustained efforts to notify, and engage diverse individuals in such opportunities for training. Furthermore, training sites ought to consider allowing less-experienced practitioners to take advantage of such training, in service of increasing diversity in the workforce. As it stands presently, many current certification programs require prior experience with the practice of meditation for at least 2 years, prior participation in residential silent retreats lasting 5 days on average, and active participation or experience teaching or receiving any type of MBAs, and often involve high costs that impede accessibility. Although these requirements are necessary to help increase the quality and depth of engagement on the part of the teacher, they can also act as an access barrier, given that, in the first place, Latinx individuals are traditionally less likely to have been on the receiving end of MBAs.
We therefore recommend that leaders and decisionmakers in the mindfulness field strategically and deliberately take steps to increase the pipeline of underrepresented individuals in the mindfulness workforce, which could include providing opportunities to meet qualification criteria (e.g., access to silent retreats that are accessible for less-experienced Latinx individuals), expanding the range to other types of contemplative practice experience such as praying, increasing the number and amount of scholarships, extending opportunities for training in mindfulness to underrepresented groups in locations where there has been low reach of mindfulness (e.g., community clinics, community organizations, non-profits, under-resourced schools, and resource centers), and training community health workers (i.e., promotores de salud), school teachers, parents, and other professionals who already interface with Latinx individuals on a day-to-day basis.

\section{Increase the Scientific Rigor of Mindfulness Research}

Recent decades have seen the proliferation of research focused on extending MBAs to minoritized groups (Castellanos et al., 2020; Cotter \& Jones, 2020). Compared to the hallmark research in this area that has relied extensively on non-Latinx individuals, the scientific literature pertaining to the application of MBAs for Latinx individuals is relatively nascent and comparatively less rigorous. In the metaanalysis conducted by Cotter and Jones (2020), many of the studies they included were characterized by small sample sizes, a lack of rigorous research designs, and a lack of longterm tracking of health-related outcomes post-intervention. The meta-analysis conducted by Castellanos et al. (2020) also noted some limitations hindering progress in this area, including a lack of detailed description regarding the adaptations made to treatment protocols and methodological designs that did not include randomization or treatment by group comparisons.

Considering these themes in the literature to date, we thus encourage researchers to increase methodological rigor of mindfulness research by specifying adaptations to mindfulness protocols (e.g., what and for whom, and why), utilizing empirically supported design methodology (e.g., multiple baseline design, randomized controlled trials), utilizing culturally relevant measurement tools, and conducting moderator analyses to answer the question of what interventions work for whom. In addition, some funding agencies at the governmental level such as the National Center for Complementary and Integrative Health $(\mathrm{NCCIH})$ have recently established funding priorities that include funding research to reduce health disparities and inequities. Thus, increasing support for early-career investigators and Latinx researchers interested in developing and increasing rigorous science and 
evidence around MBAs should be prioritized. This could be a way of increasing the pipeline and improving research skills, training, and available funding for researchers interested in conducting mindfulness research with Latinx individuals.

\section{Harness the Power of Dissemination and Implementation Science}

The field of dissemination and implementation (D\&I) science refers to the distribution of an evidence-based program or intervention to a specific audience, and to the process of translating a practice or program into a clinical or real-world setting (McHugh \& Barlow, 2010). A recent critical review of the evidence for MBAs (Dimidjian \& Segal, 2015), highlighted the need to test and develop applications of MBAs in novel populations and target problems, and to build the D\&I science around these approaches. This same study found that the majority of the reviewed studies (45\%) were classified as stage I (feasibility and pilot testing studies) or were testing the efficacy in controlled clinical trials or stage II (29\%). Less than $1 \%$ investigated efficacy in community settings (stage III), studied dissemination or implementation of MBAs (stage IV), or examined the effectiveness of MBAs in real-world settings (stage V). For MBAs to reach a population that is already largely excluded from scientific studies and existing methods of delivery, such as Latinx individuals, we recommend that researchers rely on knowledge gained from D\&I science to increase wide-scale dissemination of MBAs. However, we note that a prerequisite of wide-scale dissemination is selecting interventions that are evidencebased and have demonstrated a strong empirical base for use with a specific population. Unfortunately, this information is still limited for the use of MBAs for Latinx individuals due to the lack of representation in research combined with the lack of rigor in studies testing the effectiveness, acceptability, and feasibility of MBAs in Latinx individuals. Furthermore, the wide-scale dissemination of MBAs in Latinx individuals may require systematic and rigorous cultural adaptations of MBAs. To this point, there are likely some MBAs that, at this time, are better suited for wide-scale dissemination (such as MBSR and MBCT) given the extant strong empirical support, application to real-world settings, and use among diverse populations (Alampay et al., 2020; Gárriz et al., 2020; Michalak et al., 2020).

Moreover, in light of the new ways of connecting, interacting, and delivering programs that the COVID-19 pandemic has brought to the world, increasing research and evidence around the promising effects of online versions of MBAs should also be explored (Spijkerman et al., 2016) as this may facilitate wide-scale dissemination. Online delivery of MBAs can make important contributions to the D\&I field of MBAs, overcoming access barriers such as transportation, childcare, and other impediments usually faced by Latinx individuals.

\section{Evaluate the Safety of MBAs and Build Standards/ Governing Bodies}

While there are undoubtedly significant benefits to mindfulness practice, several recent studies have found that there are significant risks associated with a secular mindfulness practice that are often disregarded (e.g., Lindahl et al., 2019). Recently, Lindahl et al. (2017) researched the range of challenging experiences during the meditation process for practitioners in the West. The authors also explored the methods to prevent, manage, navigate, or integrate such difficult experiences for practitioners and teachers. The study found that daily practice also included reports of challenging experiences. Another study focusing on experiences of shame and exclusion in mindfulness argues that the US mindfulness corporate culture can result in, and support discriminatory acts including racism and classism which can re-traumatize and exclude Latinx individuals. Some scholars have explored the potential risks of MBAs for people with unresolved trauma, as well as the importance of trauma-sensitive approaches (e.g., Baer et al., 2019; Treleaven, 2018; Van Dam et al., 2018). Baer et al. (2019) identified several factors that need to be considered to establish the safety of MBAs namely, factors at the level of program/intervention (amount, intensity, consistency of practice; type or stage of practice), participant (psychiatric, medical, or trauma history; goals for practice, personality, health habits, relationships), and teacher/clinician (relationship with practitioner). We argue that in the case of Latinxs, having a relatable teacher who understands the process of immigration as well as acculturative and traumatic stress is imperative. Treleaven (2018) provides guidance on practicing mindfulness within one's window of tolerance by noticing one's arousal level, developing strategies for when dissociation occurs during mindfulness practice, and strategies for shifting attention to support stability, among others. Van Dam et al. (2018) note that while there have been some qualitative reports and case studies highlighting potential harms of mindfulness practice, assessments of adverse events in rigorously designed studies are still warranted to better understand potential risks associated with mindfulness practice.

To enhance the safety of MBAs for Latinx individuals, we recommend that this emerging field emulate already developed standards and governing bodies present in professional associations in health-related fields (e.g., American Medical Association [AMA, n.d.], American Psychological Association [APA, 2018], National Association of Social Workers [NASW, n.d.], and American Counseling Association [ACA, n.d.]). Several of the best-known MBAs, including MBSR and MBCT, have focused on standardization in the delivery 
of their respective programs (Santorelli, 2014; Segal, et al., 2018). In addition, three groups have made efforts to create these types of standards. First, the International Mindfulness Integrity Network (IMIN) Framework for the Integrity of Mindfulness-Based Programs (MBPs), released in 2017, seeks to "offer a comprehensive and robust set of minimal requirements for the teaching of MBPs and the training of MBP teachers" (International Mindfulness Integrity Network, n.d., p. 3). Second, the Mindfulness-Based Interventions: Teaching Assessment Criteria (MBI:TAC), a collaborative effort between the Centre for Mindfulness Research and Practice at Bangor University, the Oxford Mindfulness Centre at Oxford University, and CEDAR at Exeter University, has created criteria to "enable the teaching of Mindfulness-Based Interventions to be assessed for adherence and competence" (MBI:TAC, n.d.; Crane, et al., 2018, p. 3). Finally, the International Mindfulness Teachers Association (IMTA) was formed in 2019 by a group of well-recognized mindfulness teachers to develop and provide guidelines and to oversee national and international mindfulness instructor education and training standards (International Mindfulness Teachers Association, n.d.)

Therefore, to best protect participants, researchers, clinicians, and instructors, a regulatory structure that requires state licensure combined with a self-regulatory body that investigates and disciplines professionals who violate specified ethical mandates is recommended. To that end, the Model Practice Act for the Regulation of Secular Mindfulness Practice and Mindfulness-Based Interventions, which creates a self-regulatory organization in each state/territory governed by a Board of Mindfulness Professionals, is one possible approach (Falick, 2020). A second recommendation, as suggested by the Oxford Mindfulness Centre (2016), is for teacher training organizations to own responsibilities in this area, and collaboratively provide leadership on the "outside in" work of developing a professional context for graduates from training programs to practice within.

Careful attention should be paid to the creation of a regulatory framework within the mindfulness field, which will be critical for Latinx individuals who have sustained the type of acculturative and traumatic stressors previously discussed. The absence of researchers, clinicians, and instructors who have the training and experience in mindfulness practice to support the issues specifically faced by recent immigrants could result in significant unfavorable mindfulness experiences and constitute a failure to meet the standard of care applicable to this community.

A final consideration in order to address the root causes of many of the traumatic experiences by Latinx individuals in the US is the balance between contemplative practices and social change and activism that has been noted by Yellow Bird et al. (2020). While inner work does contribute to the larger transformation of society, it is imperative to recognize and understand the structural inequalities and social policies that undermine the health and social and economic mobility of the Latinx immigrant community in the US, and work to wisely change them.

Acknowledgements Dr. Gabriela A. Nagy and Dr. Diana C. Parra are fellows with the Research in Implementation Science for Equity (RISE), at the University of California San Francisco's Center for Vulnerable Populations; through an award from the National Heart, Lung, and Blood Institute (5R25HL126146-07). Dr. Nagy and Dr. Jennifer Plumb Vilardaga are awardees of an institutional Career Development Award through the Duke University REACH Equity Center; funded through the National Institute on Minority Health and Health Disparities (5U54MD012530-04). Additionally, Dr. Nagy is supported by a Diversity Supplement from the National Institute on Minority Health and Health Disparities (R01MD012249-03S1) and a K12 award from the Dissemination and Implementation Science in Cardiovascular Outcomes (DISCO) Program within the Department of Population Health Sciences at Duke University, funded by the National Heart, Lung, and Blood Institute (5K12HL138030).

Author Contribution GN: designed and wrote the paper. CC: designed and wrote the paper. EYRR: designed and wrote the paper. JPV: designed and wrote the paper. NZ: designed and wrote the paper. JLW: designed and wrote the paper. MCF: significantly edited the paper. DCP: significantly edited the paper.

\section{Declarations}

Conflict of Interest The authors declare no competing interests.

\section{References}

Ackert, E., Hong, S. H., Martinez, J., Van Praag, G., Aristizabal, P., \& Crosnoe, R. (2021). Understanding the health landscapes where Latinx immigrants establish residence in the US: Study examines the diversity of health contexts in which members of the US Latinx population establish residence. Health Affairs, 40(7), 1108-1116. https://doi.org/10.1377/hlthaff.2021.00176

Alampay, L. P., Tan, L. J. T. G., Tuliao, A. P., Baranek, P., Ofreneo, M. A., Lopez, G. D., ... \& Guintu, V. (2020). A pilot randomized controlled trial of a mindfulness program for Filipino children. Mindfulness, 11 (2), 303-316. https://doi.org/10.1007/ s12671-019-01124-8

Alegria, M., Canino, G., Stinson, F. S., \& Grant, B. F. (2006). Nativity and DSM-IV psychiatric disorders among Puerto Ricans, Cuban Americans, and non-Latino Whites in the United States: Results from the national epidemiologic survey on alcohol and related conditions. The Journal of Clinical Psychiatry, 67 (1), 0-0. https://doi.org/10.4088/JCP.v67n0109

Alvarez, J., Jason, L. A., Olson, B. D., Ferrari, J. R., \& Davis, M. I. (2007). Substance abuse prevalence and treatment among Latinos and Latinas. Journal of Ethnicity in Substance Abuse, 6(2), 115-141. https://doi.org/10.1300/J233v06n02_08

American Counseling Association. (n.d.). State licensing of professional counselors. Retrieved December 1, 2021, from https:// www.counseling.org/knowledge-center/licensure-requiremen ts/overview-of-state-licensing-of-professional-counselors

American Medical Association. (n.d.-e). Navigating state medical licensure. Retrieved December 1, 2021, from https://www. ama-assn.org/residents-students/career-planning-resource/ navigating-state-medical-licensure 
American Psychiatric Association. (2013). Diagnostic and statistical manual of mental disorders (5th ed.). Author.

American Psychological Association. (2018). Ethics committee rules and procedures. Retrieved December 1, 2021, from https:// www.apa.org/ethics/committee-rules-procedures-2018.pdf.

Baer, R., Crane, C., Miller, E., \& Kuyken, W. (2019). Doing no harm in mindfulness-based programs: Conceptual issues and empirical findings. Clinical Psychology Review, 71, 101-114. https://doi.org/10.1016/j.cpr.2019.01.001

Benish, S. G., Quintana, S., \& Wampold, B. E. (2011). Culturally adapted psychotherapy and the legitimacy of myth: A directcomparison meta-analysis. Journal of Counseling Psychology, 58(3), 279. https://doi.org/10.1037/a0023626

Berry, J. W. (2003). Conceptual approaches to acculturation. American Psychological Association. https://doi.org/10.1037/ 10472-004

Berry, J. W., \& Kim, U. (1988). Acculturation and mental health. In P. R. Dasen, J. W. Berry, \& N. Sartorius (Eds.), Health and crosscultural psychology. Newbury Park, CA: Sage.

Bisson, J. I., Berliner, L., Cloitre, M., Forbes, D., Jensen, T. K., Lewis, C., ... \& Shapiro, F. (2019). The international society for traumatic stress studies new guidelines for the prevention and treatment of posttraumatic stress disorder: Methodology and development process. Journal of Traumatic Stress, 32 (4), 475-483. https://doi.org/10.1002/jts.22421

Black, D. S., \& Slavich, G. M. (2016). Mindfulness meditation and the immune system: A systematic review of randomized controlled trials. Annals of the New York Academy of Sciences, 1373(1), 13-24. https://doi.org/10.1111/nyas.12998

Boyd, J. E., Lanius, R. A., \& McKinnon, M. C. (2018). Mindfulnessbased treatments for posttraumatic stress disorder: A review of the treatment literature and neurobiological evidence. Journal of Psychiatry \& Neuroscience: JPN, 43(1), 7-25. https://doi.org/ 10.1503/jpn. 170021

Braveman, P., \& Gruskin, S. (2003). Defining equity in health. Journal of Epidemiology \& Community Health, 57(4), 254-258. https:// doi.org/10.1136/jech.57.4.254

Bravo, L. G., Nagy, G. A., Stafford, A. M., McCabe, B. E., \& Gonzalez-Guarda, R. M. (2021). Adverse childhood experiences and depressive symptoms among young adult Hispanic immigrants: Moderating and mediating effects of distinct facets of acculturation stress. Issues in Mental Health Nursing, 1-11. https://doi. org/10.1080/01612840.2021.1972190

Bryant-Davis, T., \& Ocampo, C. (2005). Racist incident-based trauma. The Counseling Psychologist, 33(4), 479-500. https://doi.org/10. 1177/0011000005276465

Caballero, T. M., Johnson, S. B., Buchanan, C. R. M., \& DeCamp, L. R. (2017). Adverse childhood experiences among Hispanic children in immigrant families versus US-native families. Pediatrics, 140 (5). https://doi.org/10.1542/peds.2017-0297

Cabassa, L. J., \& Baumann, A. A. (2013). A two-way street: Bridging implementation science and cultural adaptations of mental health treatments. Implementation Science, 8(1), 90. https://doi.org/10. 1186/1748-5908-8-90

Cardoso, J. B., \& Thompson, S. J. (2010). Common themes of resilience among Latino immigrant families: A systematic review of the literature. Families in Society, 91(3), 257-265. https://doi. org/10.1606/1044-3894.4003

Castellanos, R., Spinel, M. Y., Phan, V., Orengo-Aguayo, R., Humphreys, K. L., \& Flory, K. (2020). A systematic review and metaanalysis of cultural adaptations of mindfulness-based interventions for Hispanic populations. Mindfulness, 11(2), 317-332. https://doi.org/10.1007/s12671-019-01210-x

Cervantes, R. C., Fisher, D. G., Padilla, A. M., \& Napper, L. E. (2016). The Hispanic stress inventory version 2: Improving the assessment of acculturation stress. Psychological Assessment, 28(5), 509. https://doi.org/10.1037/pas0000200

Cerdeña, J. P., Rivera, L. M., \& Spak, J. M. (2021). Intergenerational trauma in Latinxs: A scoping review. Social Science \& Medicine, 113662. https://doi.org/10.1016/j.socscimed.2020.113662

Chavez-Dueñas, N. Y., Adames, H. Y., Perez-Chavez, J. G., \& Salas, S. P. (2019). Healing ethno-racial trauma in Latinx immigrant communities: Cultivating hope, resistance, and action. American Psychologist, 74(1), 49-62. https://doi.org/10.1037/amp0000289

Chin, G., Anyanso, V., \& Greeson, J. (2019). Addressing diversity in mindfulness research on health: A narrative review using the ADDRESSING framework. Cooper Rowan Medical Journal, 1 (1). https://doi.org/10.31986/issn.2578-3343_vol1iss1.1.2

Cho, Y., Frisbie, W. P., Hummer, R. A., \& Rogers, R. G. (2004). Nativity, duration of residence, and the health of Hispanic adults in the United States. International Migration Review, 38(1), 84-211. https://doi.org/10.1111/j.1747-7379.2004.tb00193.x

Cotter, E. W., \& Jones, N. (2020). A review of Latino/Latinx participants in mindfulness-based intervention research. Mindfulness, 11(3), 529-553. https://doi.org/10.1007/s12671-019-01266-9

Crane, R. S., Soulsby, J. G., Kuyken, W., Mark, J., Williams, G., Eames, C., Bartley, T., Cooper, L., Evans, A., Fennell, M. J. V., Gold, E., Mardula, J., \& Silverton, S. (2018). Mindfulness-based interventions teaching assessment criteria (MBI:TAC): Manual, summary and addendum. http://mbitac.bangor.ac.uk/documents/ MBITACmanualsummaryandaddendums0517.pdf

Cummings, A. M., Gonzalez-Guarda, R. M., \& Sandoval, M. F. (2013). Intimate partner violence among Hispanics: A review of the literature. Journal of Family Violence, 28(2), 153-171. https://doi. org/10.1007/s10896-012-9478-5

DeLuca, S. M., Kelman, A. R., \& Waelde, L. C. (2018). A systematic review of ethnoracial representation and cultural adaptation of mindfulness-and meditation-based interventions. Psychological Studies, 63(2), 117-129. https://doi.org/10.1007/ s12646-018-0452-Z

Dimidjian, S., \& Segal, Z. V. (2015). Prospects for a clinical science of mindfulness-based intervention. American Psychologist, 70(7), 593-620. https://doi.org/10.1037/a0039589

Do, D. P., Frank, R., Zheng, C., \& Iceland, J. (2017). Hispanic segregation and poor health: It's not just black and white. American Journal of Epidemiology, 186(8), 990-999. https://doi.org/10. 1093/aje/kwx172

Duncan, B., Hotz, V. J., \& Trejo, S. J. (2006). Hispanics in the U.S. labor market. In National Research Council (Ed.), Hispanics and the future of America (pp.228-290). Washington DC: National Academies Press.

Edwards, M., Adams, E. M., Waldo, M., Hadfield, O. D., \& Biegel, G. M. (2014). Effects of a mindfulness group on Latino adolescent students: Examining levels of perceived stress, mindfulness, self-compassion, and psychological symptoms. The Journal for Specialists in Group Work, 39(2), 145-163. https://doi.org/10. 1080/01933922.2014.891683

Estrada, A. L. (2009). Mexican Americans and historical trauma theory: A theoretical perspective. Journal of Ethnicity in Substance Abuse, 8(3), 330-340. https://doi.org/10.1080/153326409031105 00

Falick, M. (2020). Mindfulness and the need to minimize the risk of harm: A proposal to implement and enforce standards for secular mindfulness practice (Master's thesis, Lesley University, Cambridge, MA). Retrieved December 1, 2021, from https://digit alcommons.lesley.edu/cgi/viewcontent.cgi?article $=1036 \&$ conte $\mathrm{xt}=$ mindfulness theses

Forner, C. (2019). What mindfulness can learn about dissociation and what dissociation can learn from mindfulness. Journal of Trauma \& Dissociation, 20(1), 1-15. https://doi.org/10.1080/15299732. 2018.1502568 
Gallegos, A. M., Crean, H. F., Pigeon, W. R., \& Heffner, K. L. (2017). Meditation and yoga for posttraumatic stress disorder: A metaanalytic review of randomized controlled trials. Clinical Psychology Review, 58, 115-124. https://doi.org/10.1016/j.cpr.2017.10. 004

Gallo, L. C., Penedo, F. J., \& Espinosa de los Monteros, K., \& Arguelles, W. (2009). Resiliency in the face of disadvantage: Do Hispanic cultural characteristics protect health outcomes? Journal of Personality, 77(6), 1707-1746. https://doi.org/10. 1111/j.1467-6494.2009.00598.x

Gany, F., Novo, P., Dobslaw, R., \& Leng, J. (2014). Urban occupational health in the Mexican and Latino/Latina immigrant population: A literature review. Journal of Immigrant and Minority Health, 16(5), 846-855. https://doi.org/10.1007/s10903-013-9806-8

García, A. A., Zuñiga, J. A., \& Lagon, C. (2017). A personal touch: The most important strategy for recruiting Latino research participants. Journal of the Transcultural Nursing Society, 28(4), 342-347. https://doi.org/10.1177/1043659616644958

Gárriz, M., Elices, M., Peretó, M., Martín-López, L. M., Justicia, A., \& Pérez, V. (2020). Mindfulness-based cognitive therapy delivered in primary care: A naturalistic, mixed-methods study of participant characteristics and experiences. Mindfulness, 11(2), 291-302. https://doi.org/10.1007/s12671-019-01166-y

Gerhart, J. I., Canetti, D., \& Hobfoll, S. E. (2015). Traumatic stress in overview: Definition, context, scope, and long-term outcomes. In Traumatic stress and long-term recovery (pp. 3-24). Springer, Cham. https://doi.org/10.1007/978-3-319-18866-9_1

Goleman, D. (2003). Destructive emotions: A dialogue with the Dalai Lama. Renaissance House Publishers.

Gonzalez-Guarda, R. M., Stafford, A. M., Nagy, G. A., Befus, D. R., \& Conklin, J. L. (2021). A systematic review of physical health consequences and acculturation stress among Latinx individuals in the United States. Biological Research for Nursing, 23(3), 362-374. https://doi.org/10.1177/1099800420968889

Goyal, M., Singh, S., Sibinga, E. M. S., Gould, N. F., Rowland-Seymour, A., Sharma, R., et al. (2014). Meditation programs for psychological stress and well-being. Journal of the American Medical Association, 174, 357-368. https://doi.org/10.1001/ jamainternmed.2013.13018

Grossman, P., \& Van Dam, N. T. (2011). Mindfulness, by any other name...: Trials and tribulations of sati in Western psychology and science. Contemporary Buddhism, 12(1), 219-239. https:// doi.org/10.1080/14639947.2011.564841

Guarnaccia, P. J., Martinez, I., \& Acosta, H. (2005). Chapter 2. Mental health in the Hispanic immigrant community: An overview. Journal of Immigrant \& Refugee Services, 3 (1-2), 21-46. https://doi. org/10.1300/J191v03n01_02

Helms, J. E., Nicolas, G., \& Green, C. E. (2012). Racism and ethnoviolence as trauma: Enhancing professional and research training. Traumatology, 18(1), 65-74. https://doi.org/10.1177/1534765610 396728

Hilton, L., Hempel, S., Ewing, B. A., Apaydin, E., Xenakis, L., Newberry, S., Colaiaco, B., Maher, A. R., Shanman, R. M., Sorbero, M. E., \& Maglione, M. A. (2017). Mindfulness meditation for chronic pain: Systematic review and meta-analysis. Annals of Behavioral Medicine: A Publication of the Society of Behavioral Medicine, 51(2), 199-213. https://doi.org/10.1007/ s12160-016-9844-2

Hinton, D. E., Ojserkis, R. A., Jalal, B., Peou, S., \& Hofmann, S. G. (2013). Loving-kindness in the treatment of traumatized refugees and minority groups: A typology of mindfulness and the nodal network model of affect and affect regulation. Journal of Clinical Psychology, 69(8), 817-828. https://doi.org/10.1002/jclp.22017

Hofmann, S. G., \& Gómez, A. F. (2017). Mindfulness-based interventions for anxiety and depression. The Psychiatric Clinics of
North America, 40(4), 739-749. https://doi.org/10.1016/j.psc. 2017.08.008

Holden, T. M., Simon, M. A., Arnold, D. T., Halloway, V., \& Gerardin, J. (2021). Structural racism and COVID-19 response: Higher risk of exposure drives disparate COVID-19 deaths among Black and Hispanic/Latinx residents of Illinois. USA. Medrxiv. https://doi. org/10.1101/2021.08.04.21261595

Holzel, B. K., Lazar, S. W., Gard, T., Schuman-Olivier, Z., Vago, D. R., \& Ott, U. (2011). How does mindfulness meditation work? Proposing mechanisms of action from a conceptual and neural perspective. Perspectives on Psychological Science, 6(6), 537559. https://doi.org/10.1177/1745691611419671

Hopwood, T. L., \& Schutte, N. S. (2017). A meta-analytic investigation of the impact of mindfulness-based interventions on post traumatic stress. Clinical Psychology Review, 57, 12-20. https:// doi.org/10.1016/j.cpr.2017.08.002

International Mindfulness Integrity Network. (n.d.). Trainings Standards and Ethical Guidelines. Retrieved December 1, 2021, from http://iminetwork.org/wp-content/uploads/2019/05/Ethics-andStandards-for-MBP_Integrity_Network.pdf

International Mindfulness Teachers Association. (n.d.). Retrieved December 1, 2021, from https://www.imta.org/page/programsta ndards

Kabat-Zinn, J. (2005). Coming to our senses: Healing ourselves and the world through mindfulness. Hachette UK.

Kearney, D. J., Malte, C. A., McManus, C., Martinez, M. E., Felleman, B., \& Simpson, T. L. (2013). Loving-kindness meditation for posttraumatic stress disorder: A pilot study. Journal of Traumatic Stress, 26, 426-434. https://doi.org/10.1002/jts.21832

Khoury, B., Sharma, M., Rush, S. E., \& Fournier, C. (2015). Mindfulness-based stress reduction for healthy individuals: A metaanalysis. Journal of Psychosomatic Research, 78(6), 519-528. https://doi.org/10.1016/j.jpsychores.2015.03.009

King, A. P., Erickson, T. M., Giardino, N. D., Favorite, T., Rauch, S. A. M., Robinson, E., . . L Liberzon, I. (2013). A pilot study of group mindfulness-based cognitive therapy (MBCT) for combat veterans with posttraumatic stress disorder (PTSD). Depression and Anxiety, 30, 638-645. https://doi.org/10.1002/da.22104

King, A. P., \& Favorite, T. K. (2016). Mindfulness-based cognitive therapy for combat-related posttraumatic stress disorder. In S. J. Eisendrath (Ed.), Mindfulness-Based Cognitive Therapy: Innovative Applications (pp. 163-189). Berlin, Germany: Springer. https://doi.org/10.1007/978-3-319-29866-5_15

Kummar, A. S. (2018). Mindfulness and fear extinction: A brief review of its current neuropsychological literature and possible implications for posttraumatic stress disorder. Psychological Reports, 121(5), 792-814. https://doi.org/10.1177/0033294117740137

Lang, A. J., Strauss, J. L., Bomyea, J., Bormann, J. E., Hickman, S. D., Good, R. C., \& Essex, M. (2012). The theoretical and empirical basis for meditation as an intervention for PTSD. Behavior Modification, 36(6), 759-786. https://doi.org/10.1177/01454 45512441200

Lehrner, A., \& Yehuda, R. (2018). Cultural trauma and epigenetic inheritance. Development and Psychopathology, 30(5), 17631777. https://doi.org/10.1017/S0954579418001153

Lin, L., Stamm, K., \& Christidis, P. (2018). How diverse is the psychology workforce? News from APA's Center for Workforce Studies. Retrieved December 1, 2021, from: https://www.apa.org/monit or/2018/02/datapoint\#: :text=Comment\%3A,from\%20other\% 20racial\%2Fethnic\%20groups.

Lindahl, J. R., Britton, W. B., Cooper, D. J., \& Kirmayer, L. J. (2019). Challenging and adverse meditation experiences: Toward a person-centered approach. In M. Farias, M. Lalljee, \& D. Brazier (Eds.), The Oxford handbook of meditation (pp. 1-26). Oxford University Press. 
Lindahl, J. R., Fisher, N. E., Cooper, D. J., Rosen, R. K., \& Britton, W. B. (2017). The varieties of contemplative experience: A mixedmethods study of meditation-related challenges in Western Buddhists. PLoS ONE, 12(5), 1-38. https://doi.org/10.1371/journal. pone. 0176239

Lopez, M. H., Gonzalez-Barrera, A., \& Krogstad, J. M. (2018). More Latinos have serious concerns about their place in America under Trump. Pew Research Center. Retrieved December 1, 2021, from: https://www.pewresearch.org/hispanic/2018/10/25/ more-latinos-have-serious-concerns-about-their-place-in-ameri ca-under-trump/

Lopez-Maya, E., Olmstead, R., \& Irwin, M. R. (2019). Mindfulness meditation and improvement in depressive symptoms among Spanish-and English speaking adults: A randomized, controlled, comparative efficacy trial. PLoS ONE, 14(7), e0219425. https:// doi.org/10.1371/journal.pone.0219425

Macias Gil, R., Marcelin, J. R., Zuniga-Blanco, B., Marquez, C., Mathew, T., \& Piggott, D. A. (2020). COVID-19 pandemic: Disparate health impact on the Hispanic/Latinx population in the United States. The Journal of Infectious Diseases, 222(10), 1592-1595. https://doi.org/10.1093/infdis/jiaa474

MBI:TAC. (n.d.). Bangor University. http://mbitac.bangor.ac.uk/mbitac-tool.php.en

Meyer, E. C., Walser, R., Hermann, B., La Bash, H., DeBeer, B. B., Morissette, S. B., et al. (2018). Acceptance and commitment therapy for co-occurring posttraumatic stress disorder and alcohol use disorders in veterans: Pilot treatment outcomes. Journal of Traumatic Stress, 31(5), 781-789. https://doi.org/10.1002/jts. 22322

Michalak, J., Mander, J., \& Heidenreich, T. (2020). Implementation and dissemination of mindfulness-based interventions. Mindfulness, 11, 275-278. https://doi.org/10.1007/s12671-019-01250-3

Mier, N., Medina, A. A., Bocanegra-Alonso, A., Castillo-Ruiz, O., Acosta-Gonzalez, R. I., \& Ramirez, J. A. (2006). Finding respondents from minority groups. Journal of Research Practice, 2(2), 1-11.

Mindfulness-Based Professional Training Institute (2020). MBSR teacher training for diverse and underserved communities. Retrieved December 1, 2021, from https://mbpti.org/mbsrttidiverse-communities/

McHugh, R. K., \& Barlow, D. H. (2010). The dissemination and implementation of evidence-based psychological treatments: A review of current efforts. American Psychologist, 65(2), 73-84. https:// doi.org/10.1037/a0018121

Nápoles-Springer, A. M., Grumbach, K., Alexander, M., Moreno-John, G., Forté, D., Rangel-Lugo, M., \& Pérez-Stable, E. J. (2000). Clinical research with older African Americans and Latinos: Perspectives from the community. Research on Aging, 22(6), 668-691. https://doi.org/10.1177/0164027500226004

National Association of Social Workers. (n.d.). https://www.socialwork ers.org/

Neece, C. L. (2014). Mindfulness-based stress reduction for parents of young children with developmental delays: Implications for parental mental health and child behavior problems. Journal of Applied Research in Intellectual Disabilities, 27(2), 174-186. https://doi.org/10.1111/jar.12064

Noe-Bustamante, L. \& Flores, A. (2019, September 16). Facts of Latinos in America. Pew Research Center. https://www.pewresearch. org/hispanic/fact-sheet/latinos-in-the-u-s-fact-sheet/

Olano, H. A., Kachan, D., Tannenbaum, S. L., Mehta, A., Annane, D., \& Lee, D. J. (2015). Engagement in mindfulness practices by US adults: Sociodemographic barriers. The Journal of Alternative and Complementary Medicine, 21 (2), 100-102. https://doi.org/ 10.1089/acm.2014.0269

Ortiz, J. A., Smith, B. W., Shelley, B. M., \& Erickson, K. S. (2019). Adapting mindfulness to engage Latinos and improve mental health in primary care: A pilot study. Mindfulness, 10(12), 2522 2531. https://doi.org/10.1007/s12671-019-01229-0

Oxford Mindfulness Centre. (October 2016). Oxfordmindfulness.org. Retrieved December 1, 2021, from https://www.oxfordmindfulne ss.org/news/know-someone-teaching-mindfulness-course-appro priately-trained/

Passel, J. S., \& Cohn, D. V. U. S. (2008). US population projections: 2005-2050. Retrieved December 1, 2021, from https://www. issuelab.org/resources/11543/11543.pdf

Perreira, K. M., \& Ornelas, I. (2013). Painful passages: Traumatic experiences and post-traumatic stress among US Immigrant Latino adolescents and their primary caregivers. International Migration Review, 47(4), 976-1005. https://doi.org/10.1111/ imre. 12050

Polusny, M. A., Erbes, C. R., Thuras, P., Moran, A., Lamberty, G. J., Collins, R. C., . . . Lim, K. O. (2015). Mindfulness-based stress reduction for posttraumatic stress disorder among veterans: A randomized clinical trial. JAMA, 314, 456-465. https://doi.org/ 10.1001/jama.2015.8361

Ramirez, R. R., \& de la Cruz, P. (2003). The Hispanic population in the United States: March 2002 (Report No. P20-545). Washington DC: U.S. Census Bureau.

Reimers, C. (2006). Economic well-being. In M. Tienda \& F. Mitchell (Eds.), Hispanics and the future of America (pp. 291-361). National Academies Press.

Rettger, J. P., Kletter, H., \& Carrion, V. (2016). Trauma and acculturative stress. In Psychotherapy for immigrant youth (pp. 87-105). Springer, Cham. https://doi.org/10.1007/978-3-319-24693-2_5

Reyes-Rodríguez, M. L., Gulisano, M., Silva, Y., Pivarunas, B., LunaReyes, K. L., \& Bulik, C. M. (2016). "Las penas con pan duelen menos": The role of food and culture in Latinas with disordered eating behaviors. Appetite, 100, 102-109. https://doi.org/10. 1016/j.appet.2016.02.029

Roth, B., \& Stanley, T. W. (2002). Mindfulness-based stress reduction and healthcare utilization in the inner city: Preliminary findings. Alternative Therapies in Health and Medicine, 8(1), 60.

Santorelli, S. F. (2014). Mindfulness-based stress reduction (MBSR): Standards of practice. The Center for Mindfulness in Medicine, Health Care, and Society, University of Massachusetts Medical School. https://www.umassmed.edu/contentassets/24cd221488 584125835e2eddce7dbb89/mbsr_standards_of_practice_2014. pdf

Schlaudt, V. A., Suarez-Morales, L., \& Black, R. A. (2021, April). Exploring the relationship of acculturative stress and anxiety symptoms in Latino youth. In Child \& Youth Care Forum (Vol. 50, No. 2, pp. 261-276). Springer US. https://doi.org/10.1007/ s10566-020-09575-0

Segal, Z. V., Williams, M., \& Teasdale, J. (2018). Mindfulness-based cognitive therapy for depression. Guilford Publications.

Sharma, M., \& Rush, S. E. (2014). Mindfulness-based stress reduction as a stress management intervention for healthy individuals: A systematic review. Journal of Evidence-Based Complementary \& Alternative Medicine, 19(4), 271-286. https://doi.org/10.1177/ 2156587214543143

Southwick, S. M., Pietrzak, R. H., Tsai, J., Krystal, J. H., \& Charney, D. (2015). Resilience: An update. PTSD Research Quarterly, 25(4), 1-10.

Spijkerman, M. P., Pots, W. T., \& Bohlmeijer, E. T. (2016). Effectiveness of online mindfulness-based interventions in improving mental health: A review and meta-analysis of randomised controlled trials. Clinical Psychology Review, 45, 102-114. https:// doi.org/10.1016/j.cpr.2016.03.009

Thompson, R. W., Arnkoff, D. B., \& Glass, C. R. (2011). Conceptualizing mindfulness and acceptance as components of psychological resilience to trauma. Trauma, Violence, \& Abuse, 12(4), 220-235. https://doi.org/10.1177/1524838011416375 
Treleaven, D. A. (2018). Trauma-sensitive mindfulness: Practices for safe and transformative healing. WW Norton \& Company.

Van Dam, N. T., Van Vugt, M. K., Vago, D. R., Schmalzl, L., Saron, C. D., Olendzki, A., ... \& Meyer, D. E. (2018). Mind the hype: A critical evaluation and prescriptive agenda for research on mindfulness and meditation. Perspectives on Psychological Science, 13 (1), 36-61. https://doi.org/10.1177/1745691617709589

Vega, W. A., Rodriguez, M. A., \& Gruskin, E. (2009). Health disparities in the Latino population. Epidemiologic Reviews, 31(1), 99-112. https://doi.org/10.1093/epirev/mxp008

Viana, A. G., Paulus, D. J., Garza, M., Lemaire, C., Bakhshaie, J., Cardoso, J. B., ... \& Zvolensky, M. J. (2017). Rumination and PTSD symptoms among trauma-exposed Latinos in primary care: Is mindful attention helpful?. Psychiatry Research, 258, 244-249. https://doi.org/10.1016/j.psychres.2017.08.042

Vinci, C., Malkhasyan, L., Simmons, V. N., \& Correa-Fernandez, V. (2020). The relationship of mindfulness and mindfulness-related practices with alcohol use among Hispanics/Latinx. Psychiatry Research, 285, 112774. https://doi.org/10.1016/j.psychres.2020. 112774
Waelde, L. C. (2021). Mindfulness and meditation in trauma treatment: The inner resources for stress program. Guilford Publications.

Waldron, E. M., Hong, S., Moskowitz, J. T., et al. (2018). A systematic review of the demographic characteristics of participants in US-based randomized controlled trials of mindfulness-based interventions. Mindfulness, 9, 1671-1692. https://doi.org/10. 1007/s12671-018-0920-5

Yehuda, R., \& Lehrner, A. (2018). Intergenerational transmission of trauma effects: Putative role of epigenetic mechanisms. World Psychiatry, 17(3), 243-257. https://doi.org/10.1002/wps.20568

Yellow Bird, M., Gehl, M., Hatton-Bowers, H., Hicks, L. M., \& RenoSmith, D. (October 2020). Defunding mindfulness: While we sit on our cushions, systemic racism runs rampant. Retrieved December 1, 2021, from: https://www.zerotothree.org/resources/ 3715-perspectives-defunding-mindfulness-while-we-sit-on-ourcushions-systemic-racism-runs-rampant\#chapter-3056

Publisher's Note Springer Nature remains neutral with regard to jurisdictional claims in published maps and institutional affiliations. 